\title{
Topography of Cytochrome Oxidase Activity in Owl Monkey Cortex ${ }^{1}$
}

\author{
ROGER B. H. TOOTELL, ${ }^{\star, 2}$ SUSAN L. HAMILTON, ${ }^{\star}$ AND MARTIN S. SILVERMAN‡ \\ * Department of Psychology, University of California, Berkeley, California 94720 and $\ddagger$ Central Institute for the Deaf, \\ St. Louis, Missouri 63110
}

\begin{abstract}
In primate cortical tissue which has been stained for the mitochondrial enzyme cytochrome oxidase, a topographical pattern of regularly spaced blobs has been demonstrated in primary visual cortex (Hendrickson, A. E., S. P. Hunt, and J. -Y. Wu (1981) Nature 292: 605-607; Horton, J. C., and D. H. Hubel (1981) Nature 292: 762-764), and a pattern of stripes has been shown in secondary visual cortex (V2) as well (Livingstone, M. S., and D. H. Hubel (1982) Proc. Natl. Acad. Sci. U. S. A. 79: 6098-6101; Tootell, R. B. H., M. S. Silverman, E. Switkes, and R. L. De Valois (1982) Soc. Neurosci. Abstr. 8: 707). These regular cytoarchitectonic landmarks have proven extremely useful in parsing the functional and anatomical architecture of these two cortical areas. In order to look for similar landmarks in other cortical areas of a primate, we completely unfolded the cortical gray matter in the owl monkey (Aotus trivirgatus), sectioned it parallel with the flattened cortical surface, and stained the tissue for cytochrome oxidase.

Distinctive cytochrome oxidase topographies were found in about seven different cortical areas. As in other primates, area V1 is characterized by blobs and area V2 is characterized by strips. In the owl monkey, area MT is characterized by an elaborate topography of dark staining in layers 1 to 4 , interspersed with light blob-shaped regions, and partially surrounded by a dark ring. Many of these topographic inhomogeneities are also reflected in the lower layer myelination topography in MT. Visual area(s) VP/VA is characterized by an irregular or strip-like topography. In some animals, a distinctive topography can be seen in area DX, which is presumably equivalent to either area DM or DI. Primary auditory cortex stains very darkly, but the overall shape of area $A$ is quite variable and the borders are indistinct. Somatosensory area 3B stains quite darkly with sharp borders, but again the overall shape of area $3 B$ is different from that previously described.
\end{abstract}

It has been shown that there are patchy, blob-like fluctuations in the level of cytochrome oxidase activity (an enzyme involved in

Received January 15, 1985; Revised March 22, 1985;

Accepted March 25, 1985

${ }^{1}$ Special thanks to are due to Russell De Valois for extensive support and encouragement during this series of experiments, and for allowing the experiments to be done in his laboratory. John Allman, Michael Stryker, and Michael Merzenich also went out of their way to lend advice and material assistance. This work was supported by United States Public Health Service Grant EY-00014 and National Science Foundation Grant BNS 82-02275.

${ }^{2}$ To whorn correspondence should be sent, at his present address: Department of Neurobiology, Harvard Medical School, Boston, MA 02115. oxidative metabolism) throughout the upper and lower layers of primate striate cortex (Hendrickson et al., 1981; Horton and Hubel, 1981). As one might expect, there is an increased number of highly reactive mitochondria within the blob regions (Carroll and WongRiley, 1982), and the array of cytochrome oxidase blobs (also described as patches, puffs, or spots) can also be seen by staining for other mitochondrial enzymes (Horton, 1984).

Recent studies have also shown that the cytochrome oxidase blobs are anatomically coupled to a whole host of variables which are less directly related to metabolic activity. The blobs in the upper layers receive a specialized input from certain layers of the lateral geniculate nucleus (Fitzpatrick et al., 1983) and project in a highly specific way to extrastriate cortex (Livingstone and Hubel, 1984). The topography of the blobs is strictly related to the topography of glutamic acid decarboxylase levels (Henrickson et al., 1981), cholinesterase staining (Graybiel and Ragsdale, 1982; Horton, 1984), ocular dominance columns (Hendrickson et al., 1981; Horton and Hubel, 1981; Tootell et al., 1982), orientation tuning (Horton and Hubel, 1981; Tootell et al., 1982; Livingstone and Hubel, 1984), color and spatial specificity (Tootell and Silverman, 1981; Tootell et al., 1982; Livingstone and Hubel, 1984), and perhaps the intracortical transport of horseradish peroxidase (Livingstone and Hubel, 1984; but see also Rockland and Lund, 1983). These various findings have changed the whole notion of a striate cortical "module" from a theoretical construct to what is rapidly becoming a well described architectonic entity.

Similar cytochrome oxidase staining patterns have also been demonstrated in the visual area surrounding primate striate cortex, V2 (Tootell and Silverman, 1981; Livingstone and Hubel, 1982; Tootell et al., 1983a). Intriguingly, it has turned out that the regions of high cytochrome oxidase activity in area $V_{1}$ project to similar regions in V2 (Livingstone and Hubel, 1984), and the functional organization of visual information in $V_{1}$ and $V_{2}$ parallels these connectional constraints (Tootell et al., 1983a). By comparing the topographical position of the cytochrome oxidase landmarks to various electrophysiological, metabolic, and connectional measures, it has thus become possible to define the modular organization of V2, just as has been done in striate. This approach should be even more illuminating in V2 than in striate, since V2 has been comparatively unexplored. If a similar system of topographical landmarks can be demonstrated in other cortical areas, then these areas also become ripe for such an approach.

In order to look for such cortical areas, we examined cytochrome oxidase and cytoarchitectonic patterns across the whole of cortex in the owl monkey (Aotus trivirgatus). This species is the obvious choice for an initial survey such as the present one because the Aotus cortex is for the most part lissencephalic, and it has been thoroughly mapped by electrophysiological means (cf. Allman et al., 1981). We report here that in Aotus, there are not just two but, rather, at least seven cortical areas which have distinctive cytochrome oxidase topographies. Five of these are visual areas, and in 
these areas the cytochrome oxidase patterns have a periodic internal structure and relatively distinct borders. Primary somatosensory and auditory cortex are also clearly discriminable from surrounding cortical areas due to their high cytochrome oxidase activity. The enzymatic borders in these areas are more sharply defined than those produced by cytoarchitectonic stains, and they are also somewhat different from those reported previously.

This study is also the first application of a complete cortical flat mounting procedure in which each hemisphere can be fully unfolded for uniformly tangential sectioning (R. B. H. Tootell and M. S. Silverman, submitted for publication). In large scale topographical studies such as the present one, this technique has proven invaluable. A preliminary account of these data has been presented previously (Toulell el al., 1983b).

\section{Materials and Methods}

Fourteen hemispheres from seven adult owl monkeys were used in the present study. All monkeys were sacrificed with an overdose of sodium pentobarbital, perfused transcardially with $4 \%$ sucrose in a $0.1 \mathrm{M}$ phosphate buffer. The brains were then removed and the hemispheres were separated In some animals sulcal landmarks were marked with indelible ink prior to flat mounting. In each hemisphere, the pia was carefully removed near regions of acute cortical curvature, and the gray matter (plus immediately subjacent white matter) was gently tcascd apart from the remaining white matter. The brain was rinsed periodically with phosphate/sucrose perfusate during this stage to keep it from drying out. The still-unfixed tissue was laid surface down on a bed of bulfer-welled filter paper in a Petri dish, gently covered with polystyrene foam, and soaked in $2.5 \%$ glutaraldehyde plus $13 \%$ sucrose in phosphate buffer for 2 days at about $2^{\circ} \mathrm{C}$. The now-fixed, flattened tissue was then bisected in order to fit subsequent sections onto the largest conventionally available slides and was cut tangentially on a freezing microtome at $40 \mu \mathrm{m}$. Further details of the flat mounting procedure are described in another publication (R. B. H. Tootell and M. S. Silverman, submitted for publication). Sections were mounted on subbed slides and alternate sections were reacted for cytochrome oxidase using a method modified from WongRiley (1979). To increase the staining intensity, sections were first incubated in a weak cobalt chloride solution $(275 \mathrm{mg} /$ liter of cobalt chloride in a $0.05 \mathrm{M}$ Tris buffer with $10 \%$ sucrose and $0.5 \%$ dimethylsulfoxide (DMSO), $\mathrm{pH} 7.4$ ) for $10 \mathrm{~min}$. Excess cobalt chloride was rinsed out by moving sections through four successive rinses of $10 \%$ sucrose/phosphate-buffered serum (PBS) (5 $\mathrm{min}$ each). The reaction solution consisted of $50 \mathrm{mg}$ of diaminober 1 idine tetrachloride, $7.5 \mathrm{mg}$ of cytochrome $c, 2 \mathrm{mg}$ of catalase, and $0.25 \mathrm{mg}$ of DMSO/100 $\mathrm{ml}$ of $10 \%$ sucrose PBS, maintained at 37 to $41^{\circ} \mathrm{C}$ and $\mathrm{pH} 7.4$. (Further details can be found in M. S. Silverman and R. B. H. Tootell manuscript in preparation.) Sections were then dehydrated in an ascending series of alcohols followed by two changes of xylenes, and coverslipped. Alternate sections were stained for Nissl substance or myelin according to several standard recipes (Weil, Weigart, thionin, cresylecht violet, Luxol fastblue). The size of various cortical areas was calculated by printing the stained brain sections on photographic paper (llford) and by cutting out and weighing the areas of interest.

\section{Results}

Figure $1 A$ shows an overall view of the cytochrome oxidase patterns in one hemisphere which has been reacted for cytochrome oxidase. To orient the reader to the flattened topography, the electrophysiological areas mapped in previous studies have been redrawn as they would appear in a flattened brain in Figure 1B. At this cortical depth (layer 4 in most areas), topographic enzyme inhomogeneities can be seen in V1 (striate), V2, (VA/VP, MT, A1, parts of area $3 \mathrm{~b}$, and the parahippocampal gyrus. In this particular case, area DX is not readily apparent, but its approximate position in the brain has been extrapolated from other cases and sketched in Figure 1B. Adjacent sections stained for Nissl substance (Fig. 1C) show fewer areas at a lower contrast and in much less detail. In those cortical areas which have a distinctive cytoarchitectonic morphology; the extent and topography of the cytochrome oxidase architecture can be usefully defined relative to the known anatomical boundarios. For clarity, topographical patterns in each of the cortical areas will be described separately.
Striate cortex (V1). Striate cortex is the largest known cortical area in the owl monkey: its surface is about $400 \mathrm{~mm}^{2}$, or $17 \%$ of the total cortical surface area (about 2300 to $2500 \mathrm{~mm}^{2}$ ). The cytochrome oxidase blobs here are quite prominent: the topography of the striate cortex blobs from two representative hemispheres are shown in Figure 2, $A$ and $B$. As in various other primate species, the blobs in the owl monkey are arranged in a grid-like patchwork which is roughly rectangular (and even strip-like in more peripheral regions; cf. Fig. 2). The sometimes strip-like nature of the blob array is less understandable in Aotus (which appears to lack ocular dominance columns: Kaas et al., 1976; Rowe et al., 1978) than in Macaca (in which the cytochrome oxidase blobs are positioned neatly along the center of ocular dominance strips: Hendrickson et al., 1981; Horton and Hubel, 1981; Toolell el al., 1982; Horton, 1984). We counted the number of blobs across two whole hemispheres taken from different monkeys, and arrived at figures of 1056 and 1023 blobs/ hemisphere. However, considering the unavoidable ambiguity in counting blobs which are incompletely separated from each other, it is safe to say only that there are "about a thousand" blobs in each Aotus hemisphere.

In the binocular segment, the spacing of the blobs is similar to that in the squirrel monkey and smaller species of macaques (about $430 \mu \mathrm{m})$. In more peripheral regions the blobs are consistently spaced further apart (about $580 \mu \mathrm{m}$ ) than they are in the binocular segment. This is probably not an artifact of the flat mount procedure (since the regions in question are relatively flat in vivo) but is more probably an actual expansion of the interblob spacing in the monocular temporal crescent, as has been described in the macaque (Horton, 1984).

The laminar organization of striate cortex (Kaas et al., 1976; Rowe et al., 1978) and of the striate cytochrome oxidase blobs (Horton, 1984 ) is clearly atypical in Aotus. In other primates which have been examined, the cytochrome oxidase blob array is clearly visible in al layers except layer 4: the common primate pattern in layer $4 \mathrm{~A}$ is reticulated, layer $4 \mathrm{~B}$ is uniformly light, and layer $4 \mathrm{C}$ is uniformly dark (Hendrickson et al., 1981; Horton and Hubel, 1981; Horton, 1984). in the owl monkey, however, the cytochrome oxidase blob array extends without obvious laminar transition from layer 1 through layer $4 \mathrm{Ca}$, including the upper layer 4 sublayer(s). As in other primates, the blob array in layers 5 and 6 is paler than that in the upper layers.

In layer $4 \mathrm{Ca}$ of Aotus, the blobs can also be seen (although very taintly) in adjacent sections stained for Nissl substance (see Fig. 3). Even under a microscope, it is not readily apparent whether the blob-like staining pattern is due to an increase in the number of blob cells in layer $4 \mathrm{Ca}$, or to an increased staining affinity in blob cells. At any rate, it is not a large effect. The blobs (and other regions of high cytochrome oxidase activity) are even more obvious when the tissue is stained for Luxol fast blue (cf. Tootell et al., 1983a), but in the latter case the affinity of the stain is not limited to myelin and it is not straightforward to interpret.

Area $\mathrm{V} 2$. As in other monkey species, the area surrounding striate cortex (V2) is characterized by cytochrome oxidase strips (Fig. 2, C and $D$ ). Area V2 in Aotus is relatively small (90 to $\left.100 \mathrm{~mm}^{2}\right)$ and the cytochrome oxidase strips here are spaced about 1 to $1.5 \mathrm{~mm}$ apart. The strips are much less obvious in Aotus than in the closely related Saimiri (Tootell et al., 1983a). However, in some animals the strips (and the rostral border of the strips) are unambiguous (cf. Fig. 8). The strips illustrated in Figure 2 are typical rather than best examples.

In Aotus there are about 16 to 20 strips spread evenly throughout V2. In other species (particularly Saimiri), thin strips alternate with thick strips, and this is vaguely true in Aotus as well. To the extent that the thin-thick generality is true, there are 8 to 10 strip pairs (or V2 "modules") in Aotus V2 rather than 16 to 20 of them. As in other primate species (e.g., Tootell et al., 1983a), there is a certain internal structure within the overall cytochrome oxidase strips.

In some animals, it can be demonstrated that there are no strips adjacent to the most peripheral striate cortex over a distance of 


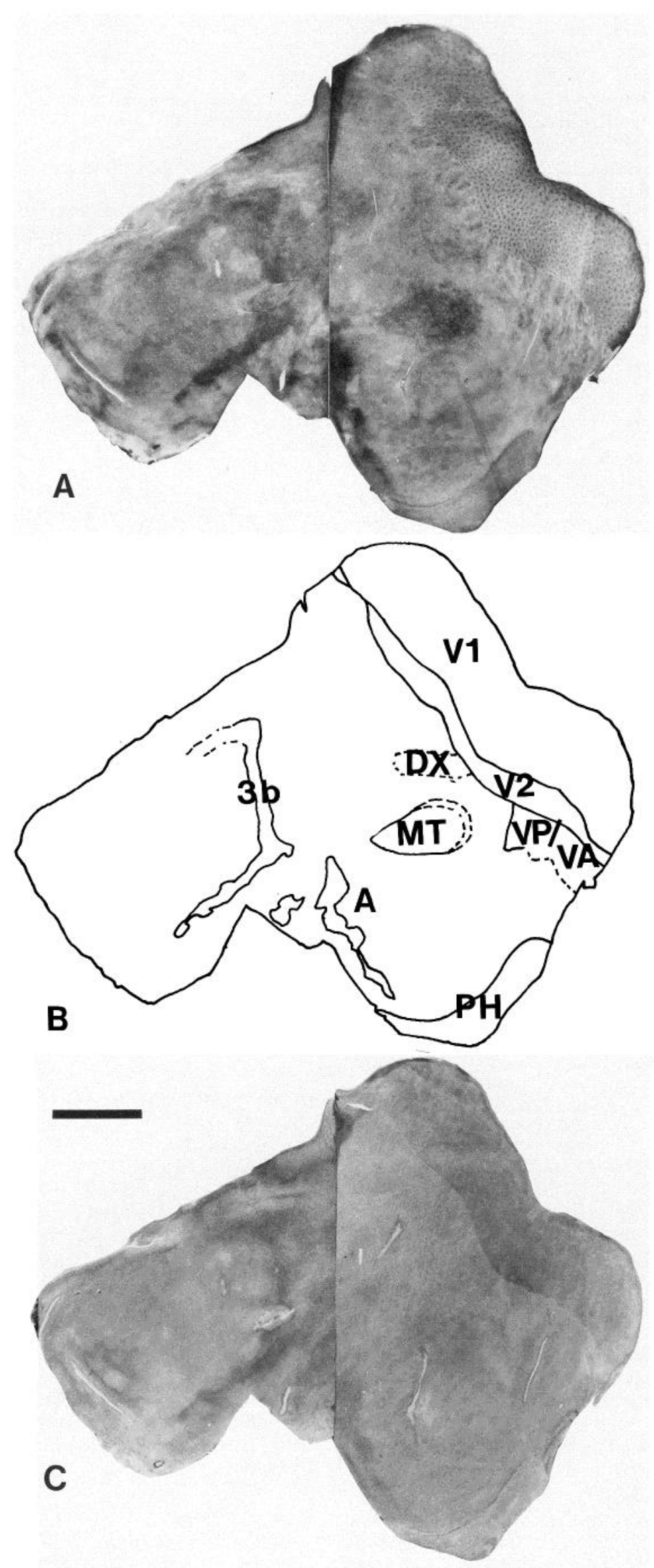

Figure 1 

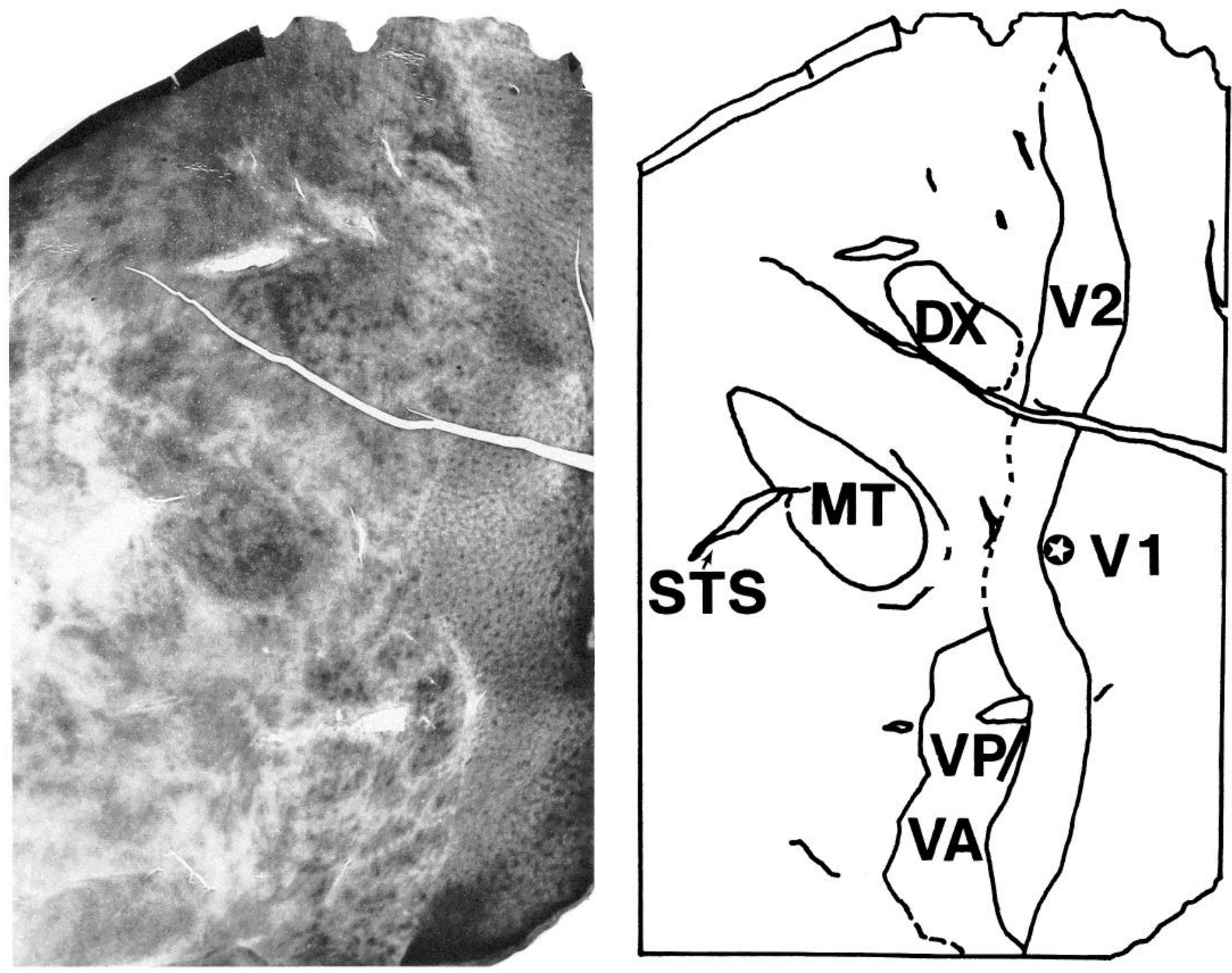

A

Figure 2. Complete cytochrome oxidase topography of $\mathrm{V} 1, \mathrm{~V} 2$, and other visual cortical areas. $A$, Portion of a flattened section including the complete caudal half of one hemisphere. The section is mostly from layer 4 , and it has been stained for cytochrome oxidase. $B$, Schematic drawing of known and estimated area boundaries in $A$. Conventions are as in Figure $1 A$. The star in the center of $V 1$ indicates the approximate position of the foveal representation. Rostral in vivo is toward the left and slightly downwards relative to the center of the figure. In $A$, the peripheral representation of V1 is uppermost in the figure, and in this region the V1 blobs are spaced further apart than those closer to the foveal representation. The contrast of the V2 strips in $A$ is significantly lower than that in some animals (e.g., the case illustrated in Fig. 9). STS, superior temporal sulcus. Calibration bar $=0.5 \mathrm{~mm}$.

Figure 1. Comparison of cytochrome oxidase, Nissl, and electrophysiological topography across all of the Aotus cortex. $A$, The complete topography of cytochrome oxidase staining patterns in Aotus cortex. Two flattened sections, mostly from layer 4, were stained for cytochrome oxidase and apposed at the straight vertical artifact near the center. Cortex which would be visible from a lateral vantage point (relative to the brain) in vivo is found near the center in $A$ to $C$. Also, at the center of $A$, axes which are rostral in vivo extend left and downward, and dorsal in vivo is upward and to the left. $B$, Schematic drawing of known and estimated area boundaries in $A$. Boundaries have been drawn based on cytoarchitectonic criteria described previously, and on electrophysiological mapping studies. Solid lines indicate area boundaries which are relatively unambiguous, and more uncertain boundaries are denoted with dashed lines. Some boundaries which are indistinct in $A$ (but nevertheless drawn in solid lines) were confirmed by referring to other sections from the same hemisphere, in which the boundaries were more obvious. V1, striate, or primary visual cortex (also called area 17); V2, secondary visual cortex, coextensive with area 18 in some species. MT, VP, and VA have no synonyms in Aotus, although area MT is probably homologous to Zeki's area V5 in the macaque. DX indicates the approximate position of an area which in some (but not this) animals shows a distinct cytochrome oxidase topography. Area DX may be coextensive with either DI or DM. Area $A$ includes (but may extend beyond) primary auditory cortex. Area $3 b$ is primary somatosensory cortex. Calibration bar $=1 \mathrm{~cm}$. C, Composite view of the Nissl topography, composed of two sections (both adjacent to the sections used in $A$ ) which have been stained for thionin and apposed along the margin which was originally bisected. More anatomical detail is obvious in $A$ than in $1 C$. Scale as $A$ and $B$. 


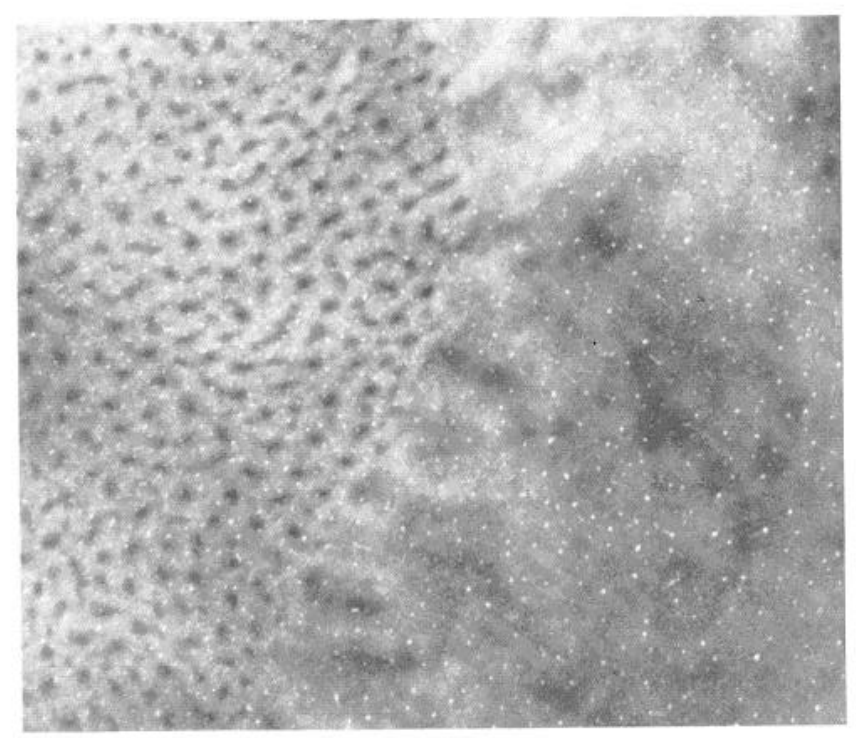

\section{A}

Figure 3. A, Close-up view of the cytochrome oxidase topography, including portions of layer $4 \mathrm{Ca}$ near the foveal $\mathrm{V} 1$-V2 border. Here $\mathrm{V} 1$ extends

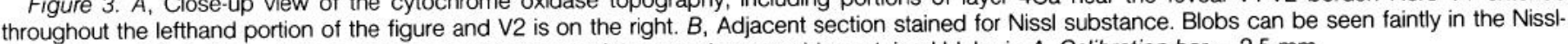
stained layer $4 \mathrm{Ca}$ in $\mathrm{V} 1$, and these match the topography of the cytochrome oxidase-stained blobs in $A$. Calibration bar $=2.5 \mathrm{~mm}$.

about $6 \mathrm{~mm}$; presumably this is an area which represents the monocular temporal crescent and the prostriate area of Sanides (Allman and Kaas, 1974a). In the macaque, the strips are confined to the binocular portion of the visual field (Tootell et al., 1983a). It is not yet clear in any species whether the area "V2" contains a complete map of both the binocular and monocular regions of the visual field, or whether it contains only a map of the binocular region.

The laminar distribution of cytochrome oxidase patterns may be atypical in Aotus V2, as it is in Aotus V1. In squirrel and macaque monkeys, the V2 strips are quite dark in the lower layers, but in the owl monkey they fade out in those same layers.

Area MT. One of the most intricate of the cytochrome oxidase topographies occurs in area MT (see Fig. 4). In layers I through 4, MT appears as a dark oval (average size, $37 \mathrm{~mm}^{2}$ ), filled with irregular/blob-like regions of lighter reaction product. The posterior portion of the oval is surrounded by a patchy ring (or more correctly an arc) of high cytochrome oxidase staining at a constant distance of about 0.7 to $1.3 \mathrm{~mm}$. The midpoint of the ring/arc is always opposite and slightly dorsal to the presumptive foveal representation of MT within the central oval. Thus, in the flattened sections, the arc is found opposite to the point where the MT oval dips into the superior temporal sulcus (see Fig. 5). Since MT has previously been described only as an oval (and not as an oval plus a ring), it is not clear whether the ring should be considered part of MT or part of DL, which surrounds most of area MT posteriorly. (However, no corresponding ring appears in the hematoxylin topography of the lower layers; see Figs. 6 and 7.)

The definition and contrast of the cytochrome oxidase topography in owl monkey MT is most obvious in layers 3 and 4; it becomes progressively fuzzier and lighter in contrast toward the cortical surface. The distinctive MT pattern disappears abruptly at the layer 4-5 border, and throughout layers 5 and 6 the cytochrome oxidase pattern in MT is uniformly light and indistinguishable from surrounding cortical areas (see Fig. 6).

Area MT is unusual among extrastriate visual areas in that it can be reliably differentiated from surrounding cortical areas by the presence of heavy myelination in the lower layers (Allman and Kaas, 1971; Van Essen et al., 1981). The distinctive heavy myelination

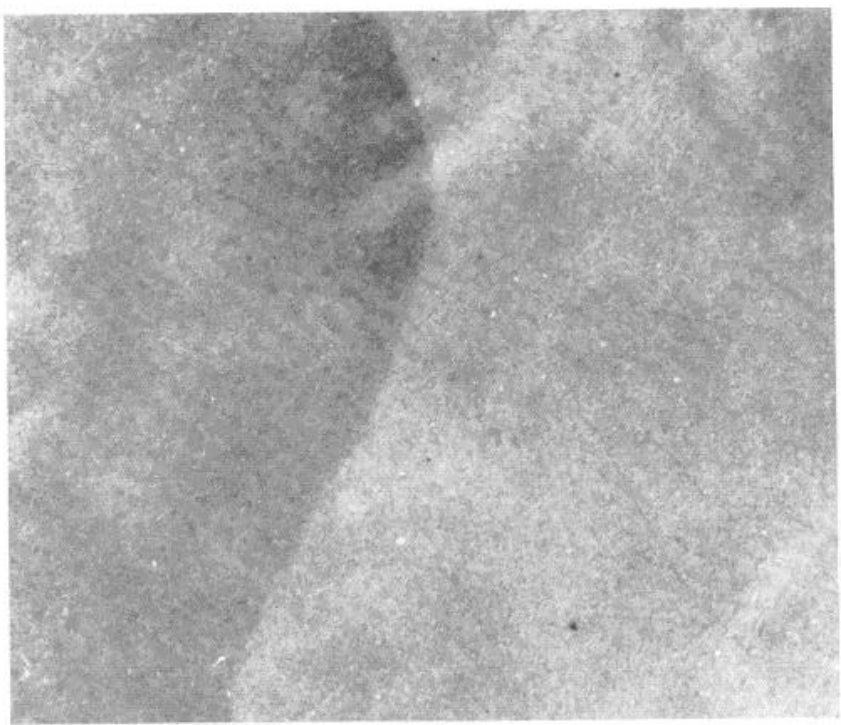

B

disappears in the upper layers of MT, whereas the dark cytochrome oxidase pattern disappears in the lower layers (see Fig. 6). Thus, the laminar distribution of heavy myelination and cytochrome oxidase activity are roughly complementary. A limitation to this generality is that the myelin patterns fade out gradually toward the upper layers, whereas the cytochrome oxidase patterns disappear abruptly at the layer 4-5 border.

If the vertical organization of the myelin and cytochrome oxidase patterns can be thought of as roughly complementary, the horizontal organization of the two patterns can be thought of as roughly identical. The heavy myelination in the lower layers is in fact topographically nonuniform, and it invariably reflects the idiosyncratic topography of the correspoinding cytochrome oxidase patterns seen in the upper and middle layers. The two patterns are positively related: regions of lighter cytochrome oxidase activity overlie regions of lighter myelination. However, the myelin pattern is much more blurred, even when the two markers are roughly equated in contrast.

In the lower layers (especially in layer 6), a trail of myelinated fibers can be seen extending anteriorly from the main body of MT in the general direction of the auditory areas (see Fig. 7). The true destination or source of these fibers and their functional significance are obscure.

Area VPIVA. So far we have described cytochrome oxidase patterns in striate and in two extrastriate areas. Both of the latter areas receive a direct, primary projection from striate cortex and are thus described as second-tier cortical areas. In this section we describe a cytochrome oxidase topography which appears to be coextensive with visual areas VP and/or VA, which do not receive a direct projection from striate (and are therefore classified as at least third-tier cortical areas).

Figure $8 A$ shows the cytochrome oxidase topography in the granular layer of area VP/VA, and Figure $8 B$ is a diagram illustrating our best estimate of corresponding electrophysiological boundaries. In many animals there are strips within VA/VP which run parallel to the adjoining V2 strips; in these animals a distinction between the V2 and VP/VA topographies can be justified by the presence of: (1) an abrupt discontinuity in the length of the cytochrome oxidase strips at the presumptive intersection of the V2, DL, and VP borders 


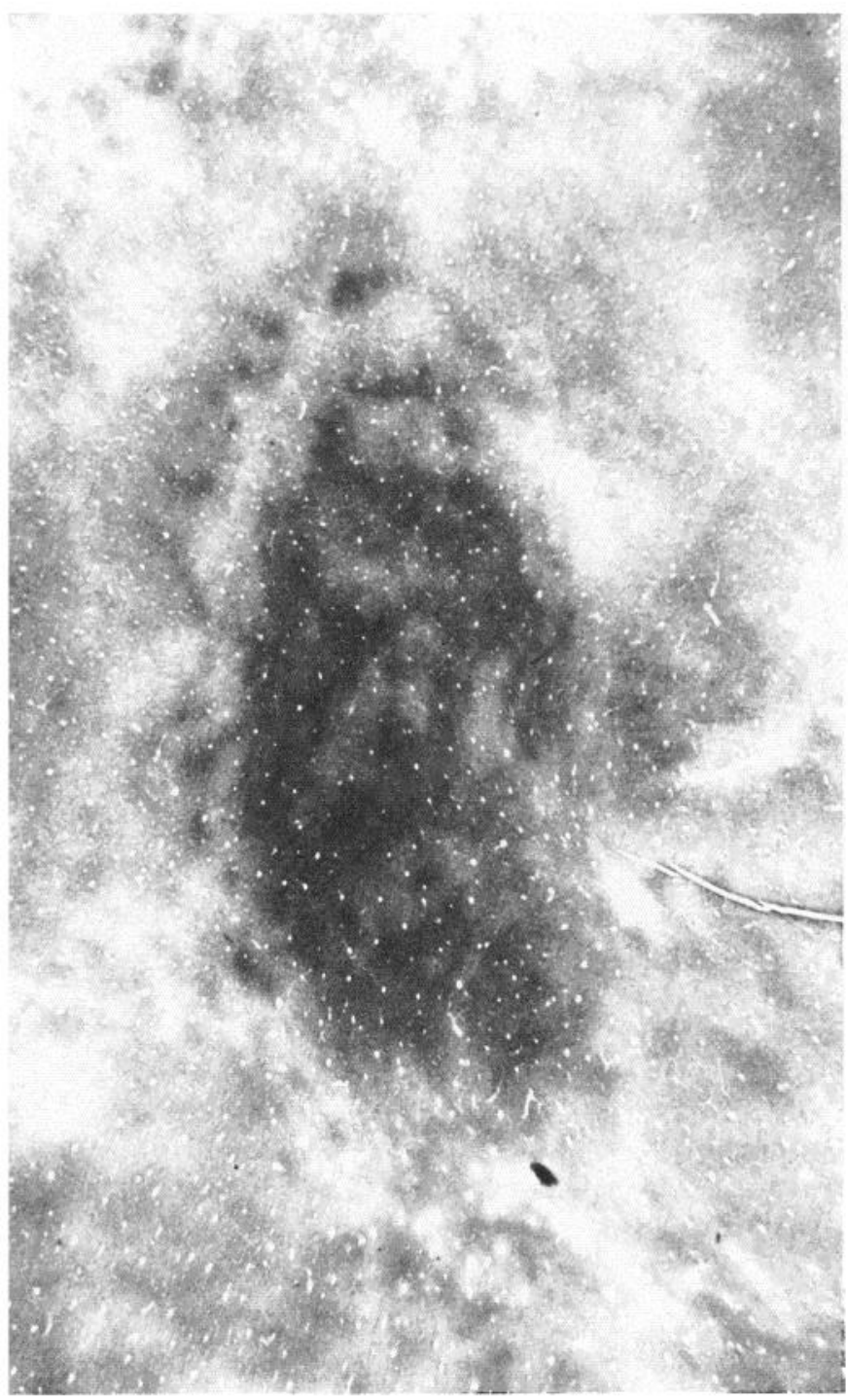

Figure 4. Close-up of the topography of cytochrome oxidase staining patterns in layer 4 of cortical area MT. Irregular, blobby areas of light staining are visible within the darkly staining central oval. In the upper left of the figure, a patchy ring of dark reaction product surrounds part of MT. Calibration bar $=2.5 \mathrm{~mm}$.

(Fig. $8 A) ;(2)$ the lack of a similar lengthening of strips adjacent to mirror-symmetrical portions of dorsal V2; (3) a characteristic break or discontinuity in the strips at the presumptive V2-VP border; and (4) a close match between the size and position of electrophysiologically defined VP/VA and the size and position of the cytochrome oxidase landmarks. In other animals the cytochrome oxidase inhomogeneities in VP/VA are not particularly strip-like, and in these animals one can be correspondingly more confident about attributing the patterns to areas beyond V2. As nearly as we can calculate, the size of area VP/VA is about $40 \mathrm{~mm}^{2}$.

The contrast of the Aotus VP/VA patterns is less robust than that in $\mathrm{MT}$, and sometimes slightly less than those in V2. In the squirrel monkey, however, an apparently homologous cytochrome oxidase topography can be quite striking (R. B. H. Tootell, M. S. Silverman, and $G$. H. Jacobs, unpublished observations).

The laminar distribution of the VP/VA patterns in the owl monkey is similar to that found in MT and V2: the contrast gradually increases with increasing cortical depth, and then disappears abruptly, in this case at the layer 3-4 border.

Area $D X$. There is one final visual area in which a distinctive cytochrome oxidase topography can be demonstrated. However, it cannot be demonstrated in every animal. Possible reasons for this variability between animals are considered under "Discussion." When a topography can be demonstrated, it appears as in Figure 9, or (less obviously) in Figure 2, $A$ and $C$. This pattern occurs near the dorsal-medial crest of the owl monkey brain, anterior to V2. This corresponds to a position near (but perhaps not identical to) electrophysiologically defined DM or DI. In order to indicate our uncertainty about the correspondence between this anatomically defined area and electropysiological areas DM and $\mathrm{DI}$, we refer to the anatomical area (provisionally) as DX.

Area DM has been described as heavily myelinated in the lower layers (Allman and Kaas, 1975), as is area MT. In one animal we were able to compare patterns of the topography of this myelination with the cytochrome oxidase patterns; the correspondence was fairly good. When it appears, the cytochrome oxidase pattern in DX has roughly the same contrast and laminar distribution as those in V2 and VP/VA

Auditory area(s): Area A. A quite striking set of cytochrome oxidase patches appears on the lateral surface of the superior temporal gyrus, extending into the depths of the lateral fissure. The shape of the topography here is quite variable, but it generally includes a central oval core, one or more small, isolated patches (on any side except posterior to the central core), and sometimes sharply pointed, radiating patterns running approximately parallel to the lateral sulcus (Fig. 10). The cytochrome oxidase staining here is invariably quite robust and the area is easy to spot even in tissue which has suffered the worst of histological insults. The topography of the central core extends from layer 1 through layer 4-5, and that of adjacent subfields is restricted to more superficial laminae. The topography is somewhat more sharply defined in layer 4-5 than in the upper layers, but the borders of the area are not particularly sharp in any layer. Gradual borders between physiologically distinct regions in this area have also been reported (Imig et al., 1977). Some internal periodicity is usually present. This takes the form of mottled, irregular regions of lighter staining within the middle layers of the larger dark regions. The internal pattern is reminiscent of that in MT, except that the lighter regions in this area are smaller.

We have called this group of cytochrome oxidase patterns "area $A$ " to designate an area which includes (but probably extends beyond) primary auditory cortex. Although owl monkey auditory cortex has already been mapped and named (Imig et al., 1977), the topographical results in the present study are sufficiently novel that a new and provisional area name is justified, at least until current ambiguities are resolved.

Evidence that the cytochrome oxidase patterns include primary auditory cortex comes from a comparison of the enzyme map to both sulcal and cytoarchitectonic landmarks. In one animal, we related the position of primary auditory cortex in the unfolded brain (as defined by Imig et al., 1977) to its position in the flat mounted tissue. This was done by making three radial penetrations in the brain in situ with an indelible ink pen, aimed at a triangulation of area A1. Subsequent staining of the tissue for cytochrome oxidase revealed a very close correspondence between these sulcal landmarks and the central core of high enzymatic activity (see Fig. 10). Deviations between the enzymatic and the ink measures are essentially insignificant. However, in other animals the overall topography of area $\mathrm{A}$ includes several clumps of dark cytochrome oxidase staining, and separate clumps are hard to reconcile with the single area of primary auditory cortex described in an earlier study (Imig et al., 1977).

In the owl monkey, auditory areas $\mathrm{A} 1$ and $\mathrm{R}$ are dominated by densely packed, small cells in layers 3,4 , and 5 . The cytoarchitec- 

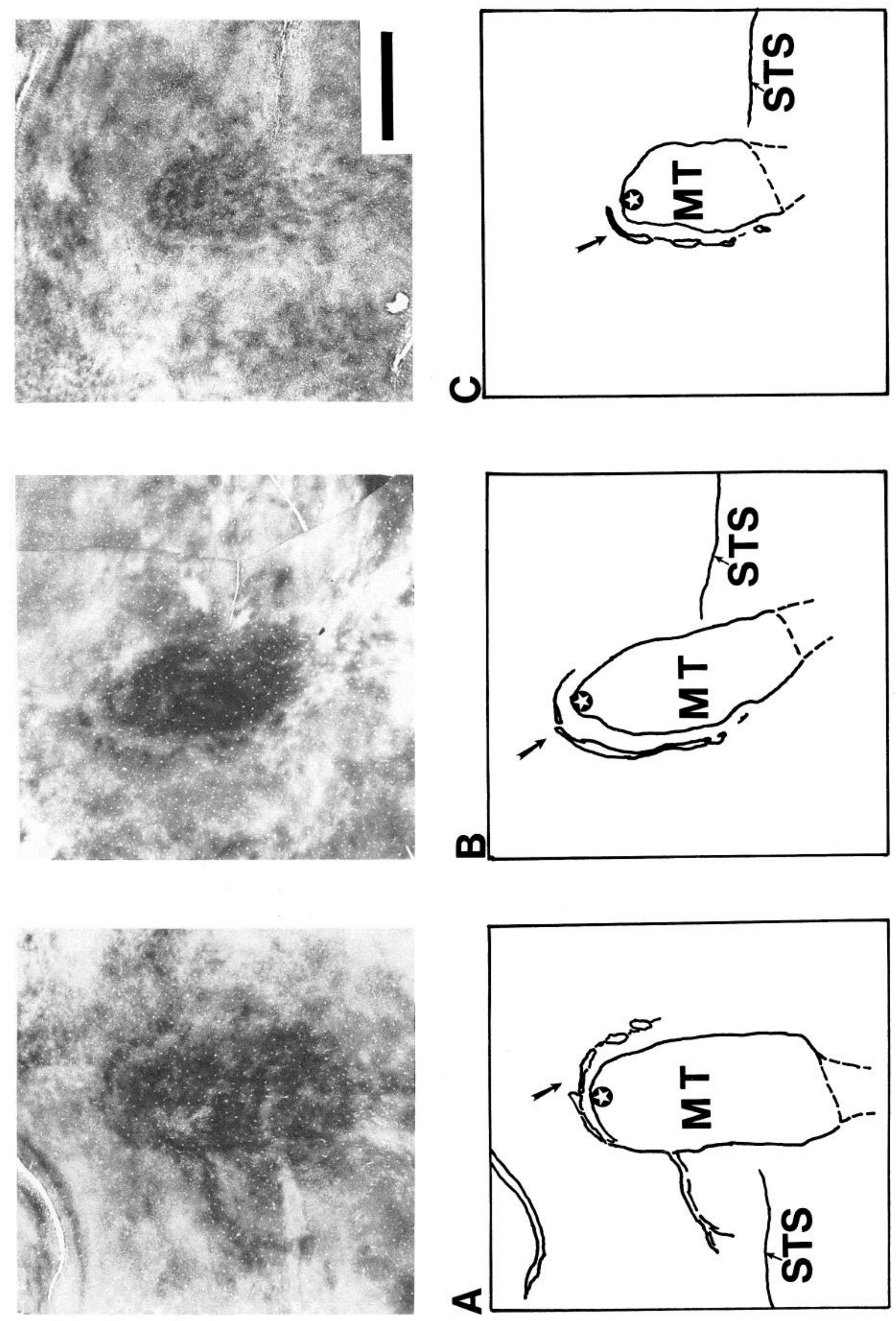

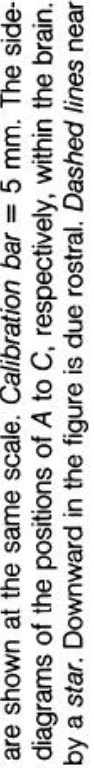

응 웛

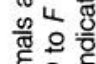

突口. 므

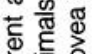

뉸

ㅎํㅇㅎㅀ

ชิ

ट्षे कू

ल ब्ष

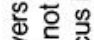

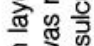

돈 $\frac{1}{0}$

등 웅

票

띤 뜬 흥

0 ठ ल

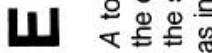

है

ธิ

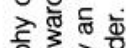

웡

응 뭉

둥

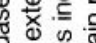

응 ํํㅇ

凹. 등

के

บ

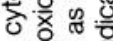

\&

든 융응

은 응

तั ले

$>$ 흐응

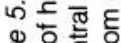

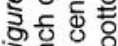

口 0 

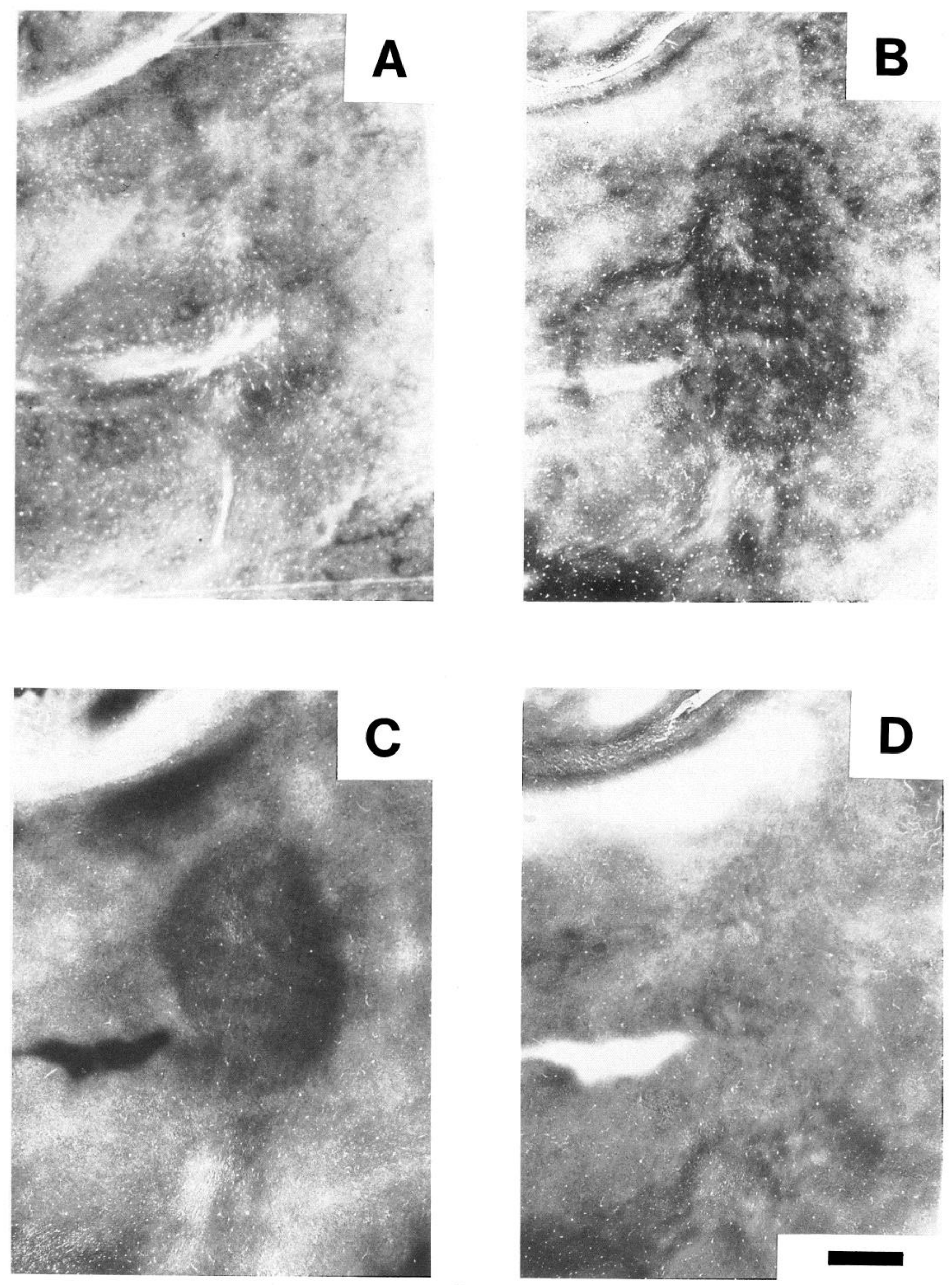

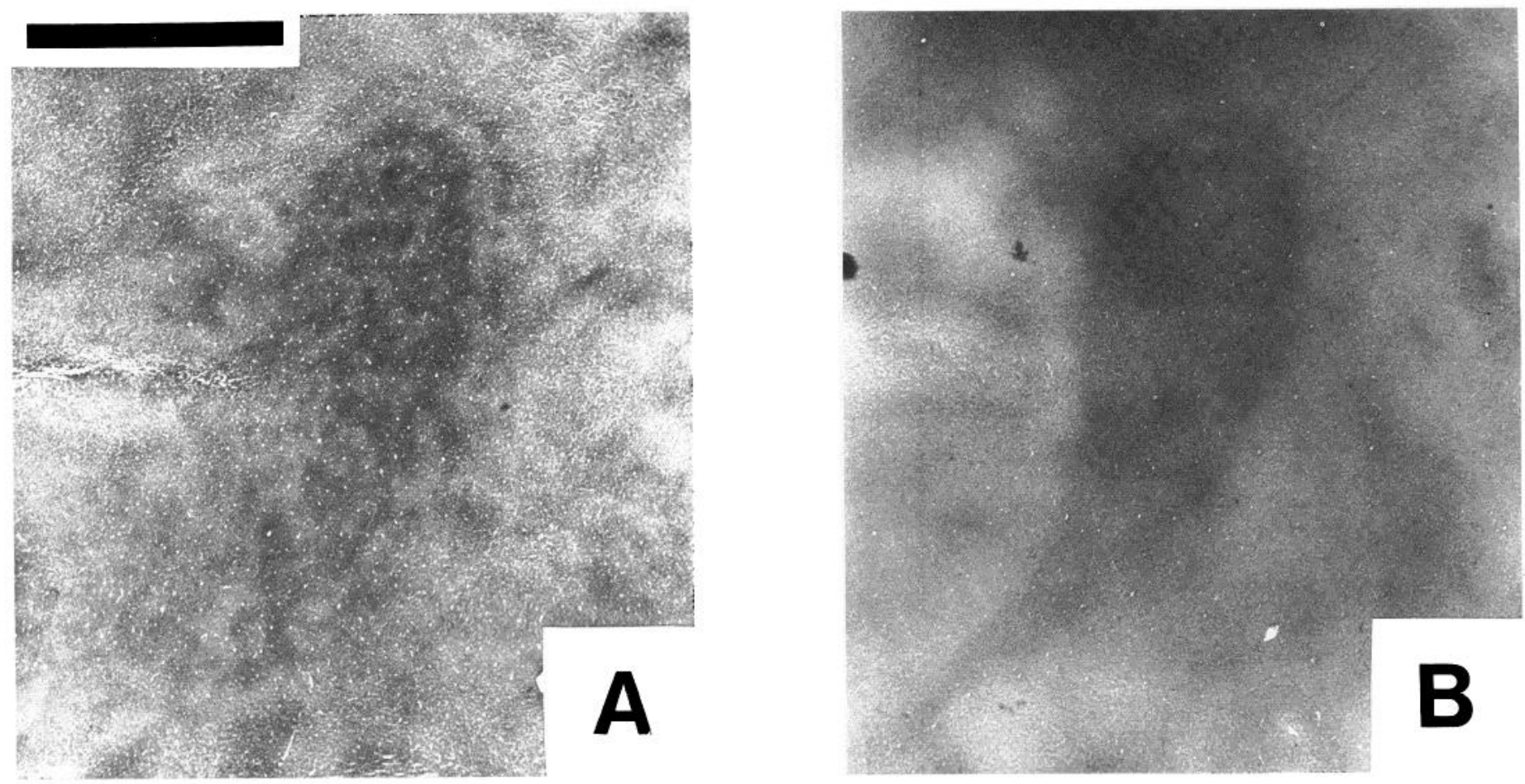

Figure 7. Close-up of a particularly prominent myelin trail which extends rostrally from MT. A shows the cytochrome oxidase topography of MT and surrounding cortex in layer 4. B shows the myelin topography from layer 6 of the same hemisphere, at the same scale. In this figure, rostral is down and toward the left. Calibration bar $=5 \mathrm{~mm}$.

tonic differentiation is obvious enough that it can be used to define A1 and R macroscopically. When we compared our Nissl-stained material to adjacent enzyme-stained sections, we found that there was in fact a good correspondence at the central core (cf. Fig. 1A). Often, however, the cytochrome oxidase patches extended beyond the areas of dark Nissl staining. It has also been reported that primary auditory cortex is more highly myelinated than surrounding areas. Comparisons between enzymatic and myelin topographies also show a good correspondence, although again the cytochrome oxidase topography is more extensive, higher in contrast, and more detailed than the myelin topography.

Area $3 b$. The final area which shows distinctive cytochrome oxidase staining is coextensive with somatosensory area $3 \mathrm{~b}$ (primary somatosensory cortex), at least along that portion of area $3 \mathrm{~b}$ which has been well mapped to date (e.g., Merzenich et al., 1978, 1981). This portion of area $3 \mathrm{~b}$ also has a distinctive Nissl stain and myeloarchitecture. The cytoarchitectonic and cytochrome oxidase topographies can be compared in Figure 1, $A$ and $C$. The well explored portion of area $3 \mathrm{~b}$ lies on the exposed lateral surface of Aotus cortex and is indicated between the arrows in Figure 11. The enzymatic and architectonic topographies are essentially identical, except that details in the enzymatic stain are more sharply defined. As in previous reports (Merzenich et al., 1978), area $3 \mathrm{~b}$ in our material runs through the central dimple (the Aotus homologue of a central sulcus).

However, it is also readily apparent that the cytochrome oxidase pattern extends beyond the well explored portion of area $3 b$, without significant alteration in width, design, staining intensity, or laminar distribution. The enzyme pattern bends sharply and continues for another 1.5 to $2 \mathrm{~cm}$ anterior to the ventral extent of the previously described region. There is a parallel (but much more diminutive) extension of the area $3 \mathrm{~b}$ strip which runs a short distance anterior from the dorsomedial extent of (previously described) area $3 \mathrm{~b}$ before gradually fading out. Thus, we find that the pattern in classic area $3 \mathrm{~b}$ merges without apparent transition into other areas which show architectural features indistinguishable from those in previously described area $3 \mathrm{~b}$. The most parsimonious solution is to expand the definition of area $3 \mathrm{~b}$ to include the whole extent of the cytochrome oxidase strip, which is what we have done. This is also in accordance with preliminary single-unit results (J. Kaas, personal communication).

We have said that our data from the well explored portion of area $3 \mathrm{~b}$ conform to previous maps of that area, but in fact this is an oversimplification: our data conform to the cytoarchitectonic and sulcal criterion for area $3 \mathrm{~b}$, but in most cases the overall topography of area $3 \mathrm{~b}$ in the present material is different from that previously described. Rather than the single dorsoventral strip wich has previously been described, in our material there were often two " $3 \mathrm{~b}$ " stripes running dorsoventrally for a distance (e.g., Fig. 11C), often connected at one or both ends (e.g., Fig. 11, $A$ and $B$ ). In these instances as well as in the single-stripe cases, both cytoarchitectonic and enzymatic stains demonstrated an identical overall topography; thus, the two-stripe results cannot be attributed to the use of different stains in the present versus previous studies. The complete strip topography includes about $60 \mathrm{~mm}^{2}$.

Within the strips there is little obvious periodicity: the density within

Figure 6. Laminar differences in myelin and cytochrome oxidase staining patterns within area MT. All figures are taken from the same topographical area of a single hemisphere. The sections illustrated in $A$ and $C$ have been stained for myelin (Weil stain), and $B$ and $D$ have been stained for cytochrome oxidase. $A$ is taken from layers $2+3, B$ is from layers $3+4$, and $C$ and $D$ are taken from layer 6 . The myelin pattern is confined to the lower layers and the cytochrome oxidase pattern is found only in the upper layers. By inspection of $B$ and $C$ it can also be shown that the regions of lighter and darker myelination in the lower layers have the same idiosyncratic topography as the cytochrome oxidase-stained sections, except that the myelin topography is much blurrier and lower in contrast. A faint myelin trail extends downward (rostral in vivo) from MT in figure C. Calibration bar $=2.5 \mathrm{~mm}$. 

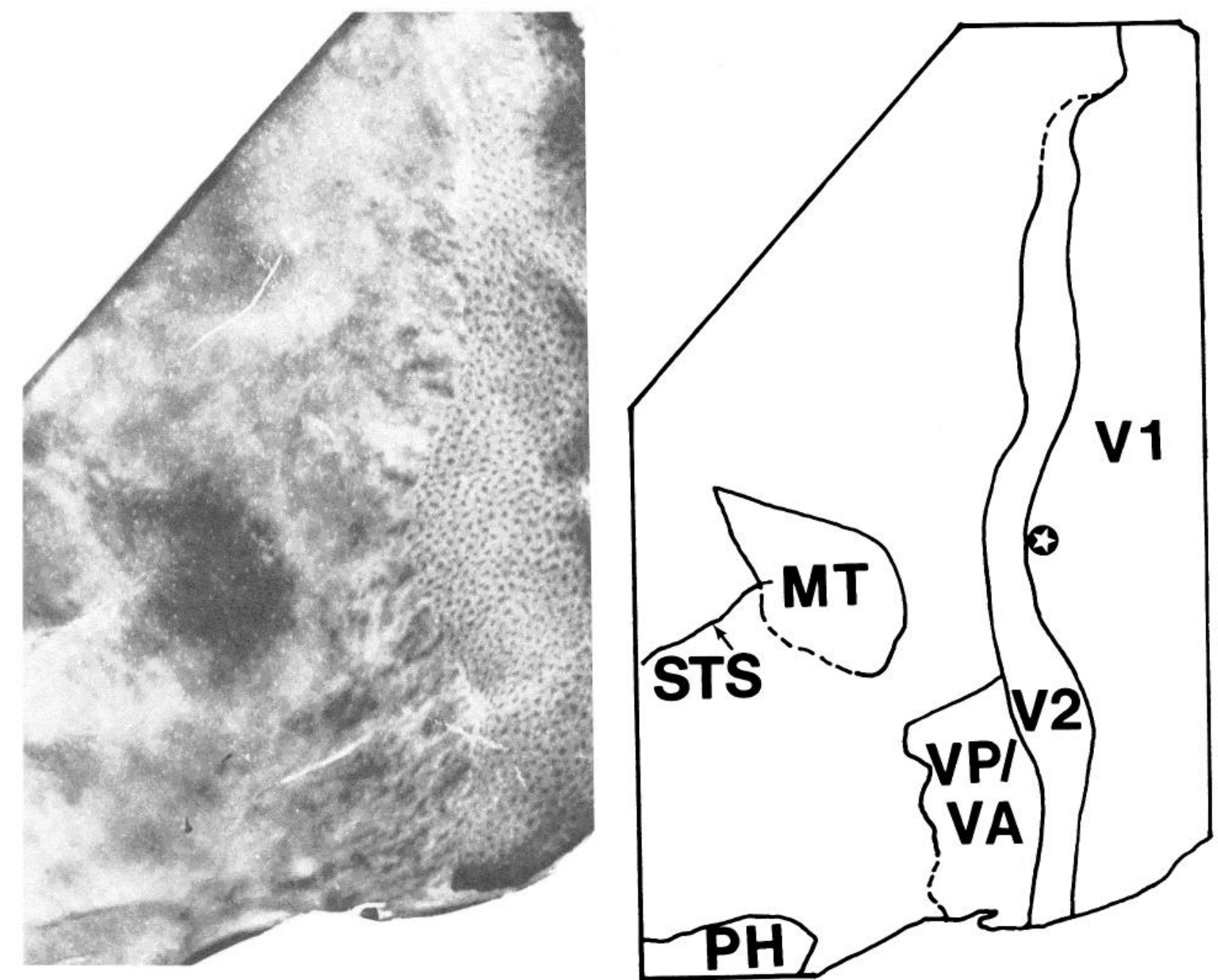

A

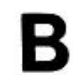

Figure 8. An example of the cytochrome oxidase topography of area VP/VA. A is a portion of a single section taken from layer $3+4$, showing VP/VA and other visual areas in the caudal half of the Aotus cortex. $B$ shows the approximate boundaries of the cortical areas shown in $A$, drawn to the same scale. Leftward in these figures is rostral in the brain. The star in V1 denotes the foveal representation. Calibration bar $=1 \mathrm{~cm}$.

the strip is fairly constant. The borders of the strips are quite sharp in the cytochrome oxidase stain and progressively less so in the Nissl and myelin stains.

The increased Nissl staining in area $3 \mathrm{~b}$ is due to the presence of densely packed granular cells which extend from layer 2 through layer 4 . The laminar distribution of the cytochrome oxidase staining in area $3 \mathrm{~B}$ is similar to that of the Nissl staining.

\section{Discussion}

The central finding of the present study is that there are about seven areas with distinctive cytochrome oxidase topography in the owl monkey cortex. In five of these areas, cytochrome oxidase patterns have not been described previously. Each area furnishes a stable architectural substrate to which connectional and physiological variables can be related in subsequent studies. The cytochrome oxidase patterns may also allow a quick comparative index of the extent, position, and existence of homologous visual areas across various primate species.

In V1 and V2, cytochrome oxidase topographies similar to those seen in Aotus have already been described in other primate species (Hendrickson et al., 1981; Horton and Hubel, 1981; Livingstone and Hubel, 1982; Tootell et al., 1983a). Relative to these species, the major difference in the architecture of these patterns in Aotus occurs in the ventrical (translaminar) rather than the horizontal (topographical) domain. Unlike the case in other primates, the Aotus VI spot array extends prominently through most of layer 4 , and the Aotus V2 strips are anomalously absent in the lower cortical layers. In this admittedly restricted comparison across species, the topography is evolutionarily more conservative than the laminar organization (and thus in some sense it may be more basic to the organization of visual information in the primate). This finding underscores the importance of studying the horizontal in addition to the vertical organization of the cerebral cortex.

In two of the visual areas, the geometry of the cytochrome oxidase topography has an interesting (and perhaps significant) relationship to the retinotopic organization of each area. For instance, in all monkey species studied so far, the V2 strips run generally perpendicular to the V1-V2 border (Tootell et al., 1983a, b). This means that each idealized strip runs from a point along the representation of the vertical meridian at the V1-V2 border to a point along the representation of the horizontal meridian at the V2-V3 border, all at a single retinal eccentricity. Thus, if each strip could be projected from its visual field representation in vivo to that portion of the visual field which each strip subserves, the pattern would look roughly like 


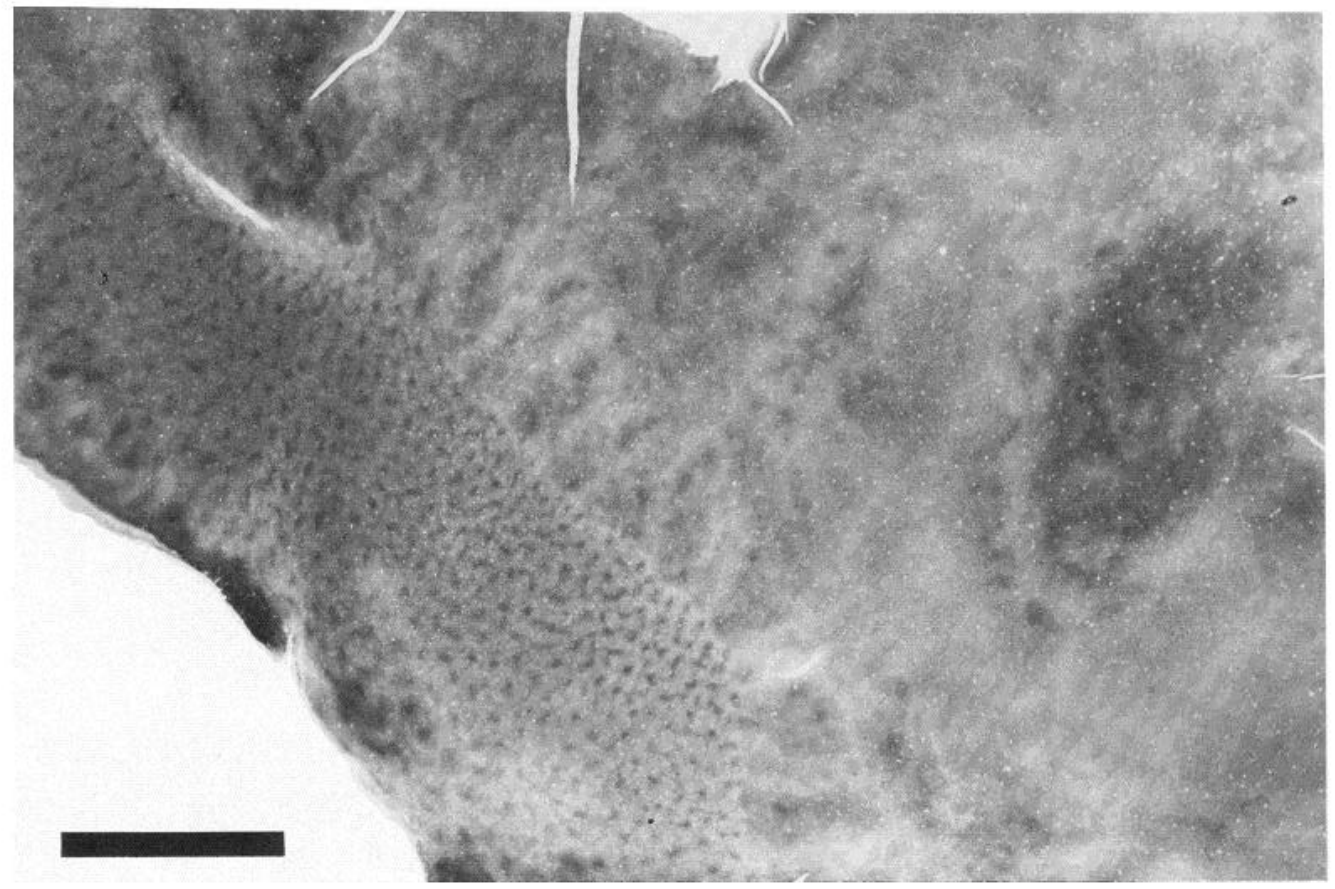

A

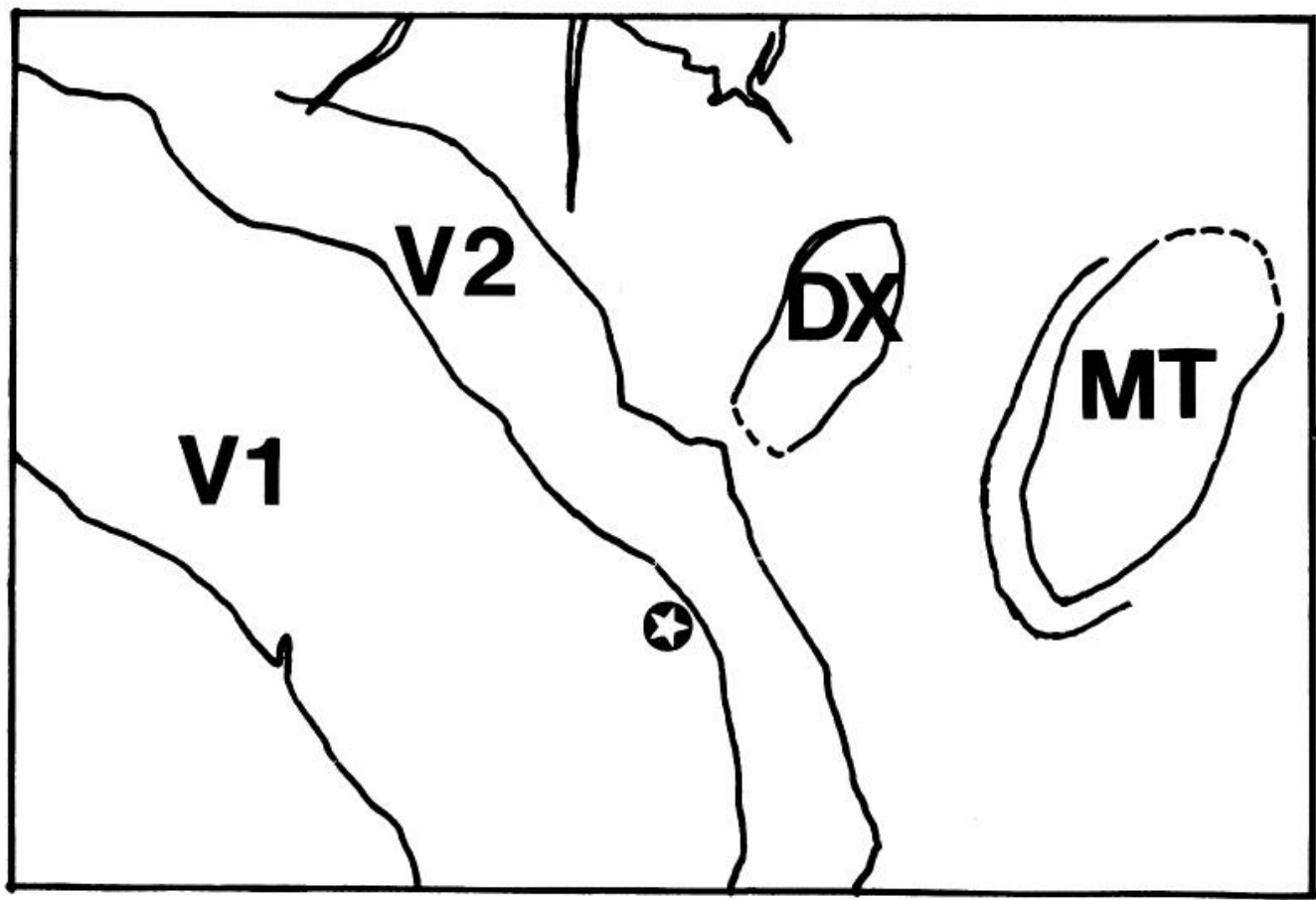

\section{B}

Figure 9. An example of the cytochrome oxidase topography of area DX. A is a portion of a single section taken from cortical layer 4 , stained for cytochrome oxidase. The section includes visual areas DX, MT, and portions of V1 and V2. B shows the approximate boundaries of cortical areas shown in $A$, at the same scale. Rostral in the brain is upward and to the right. The approximate foveal representation in V1 is indicated by the star. The V2 strips are fairly obvious in this particular case. Calibration bar $=5 \mathrm{~mm}$. 

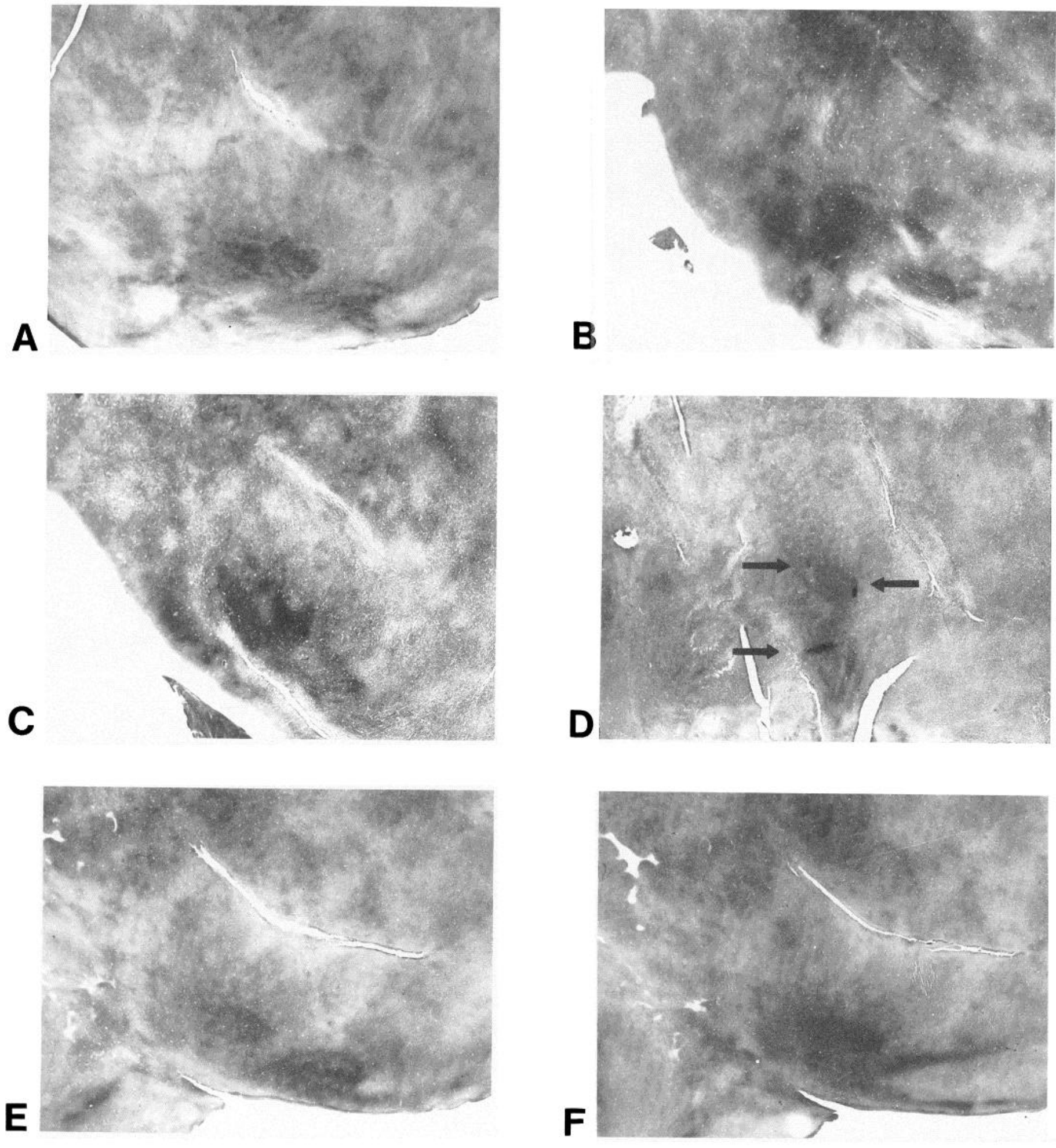

Figure 10. Variation in the topography of cytochrome oxidase patterns in auditory cortex. Each panel is a portion of a single section, stained for cytochrome oxidase and shown at the same scale (calibration bar $=5 \mathrm{~mm}$ ). Rostral in vivo is downward in each panel. The darkly staining area in the lower center of each panel includes primary (and perhaps portions of secondary) auditory cortex. $A$ to $E$ are taken from layer 3 of different hemispheres. Although substantial variation in the topography is visible between hemispheres, the internal and external topographical boundaries are usually quite blurry. $E$ and $F$ contrast laminar differences in the topography of two overlying sections from the same hemisphere. $F$ is taken from deeper layers than $E$, and at deeper layers some of the darkly staining upper layer areas drop out. $F$ also shows a pattern of sharply defined dark streaks which run parallel with the lateral sulcus. $D$ shows the cytochrome oxidase topography in an animal in which three India ink marks were made in the unstained brain in situ, triangulating the position of auditory cortex as described by Imig et al. (1977). The correspondence of the two is quite good. 
A

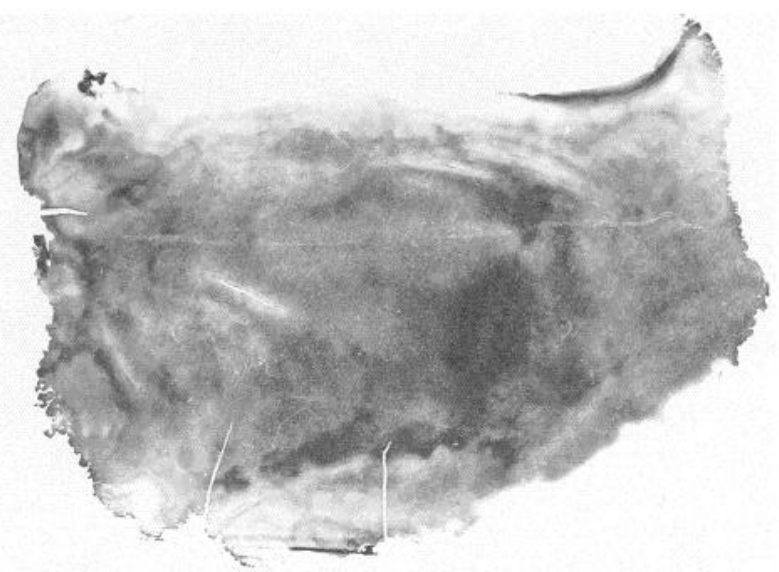

D

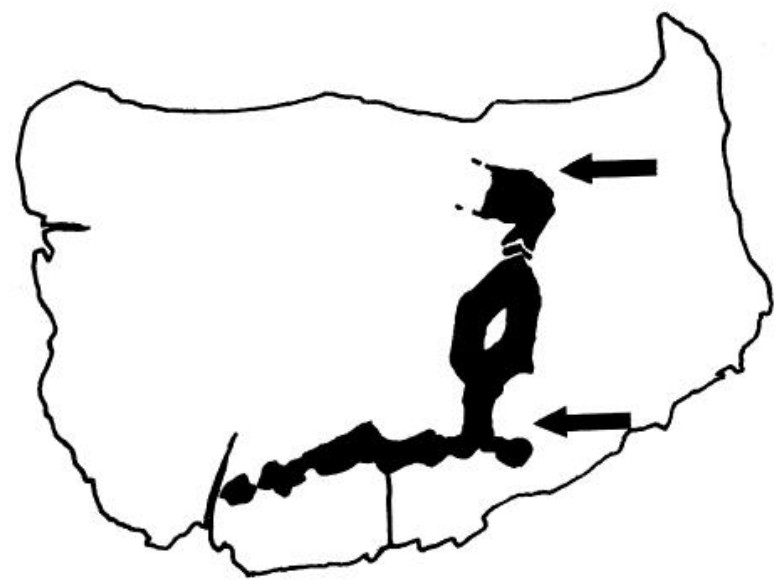

B

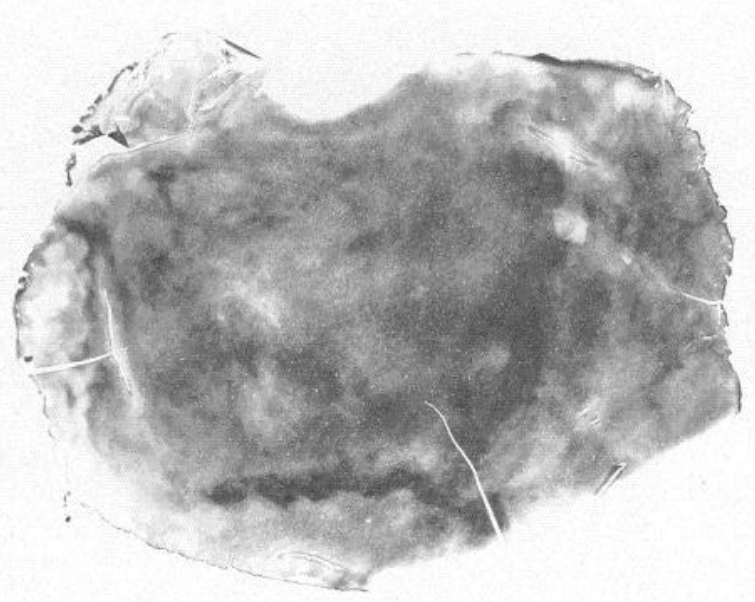

E
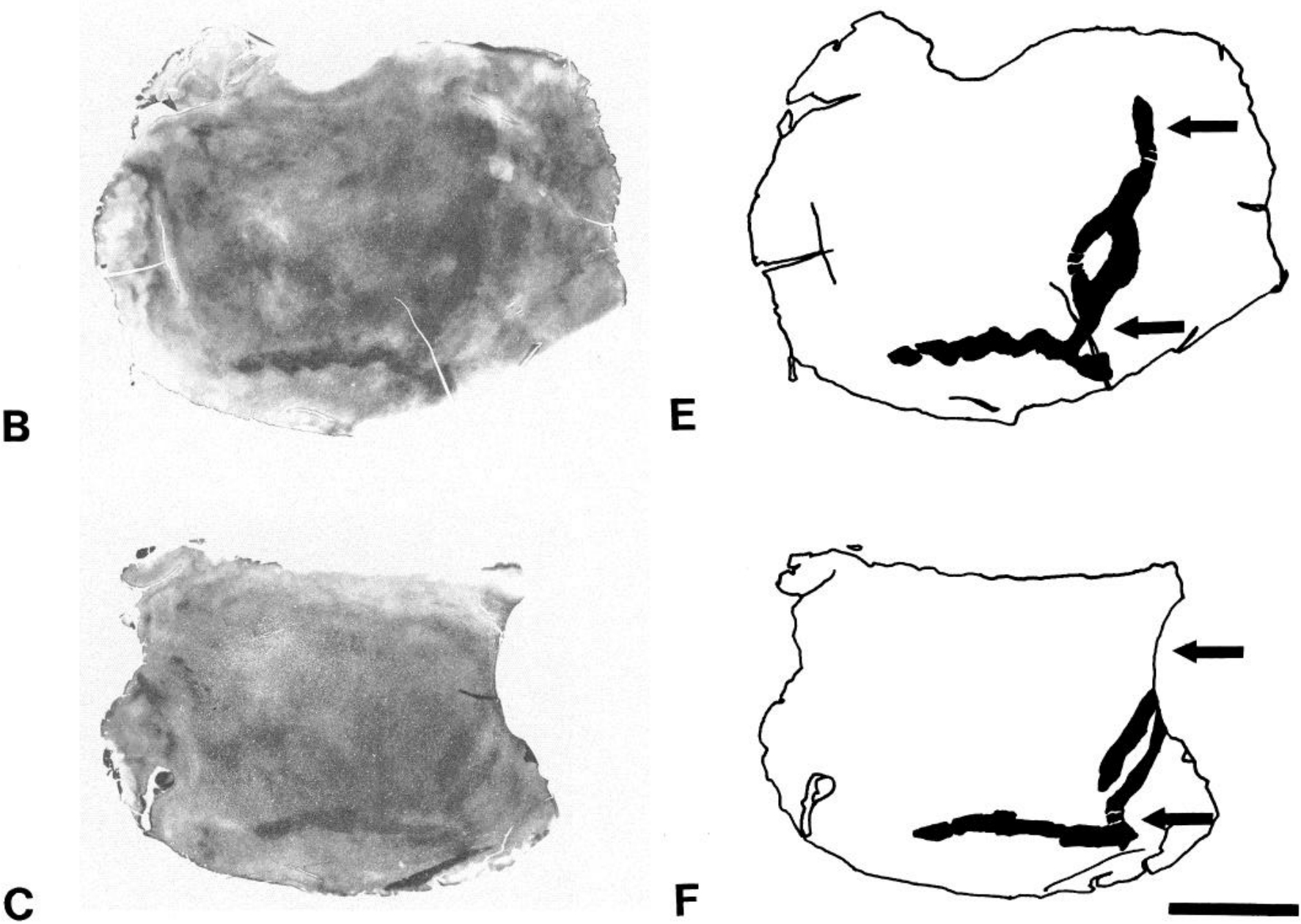

C

$\mathbf{F}$

Figure 11. Three examples of the area 3b cytochrome oxidase topography. $A$ to $C$ are single sections taken from layer 4 of three different animals, all arranged so that rostral in vivo is leftward. $D$ to $F$ are schematic drawings of $A$ to $C$, respectively, shown at the same scale. Areas of high cytochrome oxidase activity in the tissue sections (and in sections adjacent to those illustrated) are drawn in solid black in $D$ to $F$. Calibration bar $=1 \mathrm{~cm}$.

an arc of $90^{\circ}$, centered on the fixation of gaze. When the visual field representations of all the strips are combined onto a single map of the visual field, they form a set of concentric rings, expanding in size from a point at the center of gaze.

A similar relationship holds for area MT. According to the electrophysiological mapping data, the vertical meridian is represented along the outer boundary of MT, and the center of gaze is represented at the posterior aspect. Thus, if the MT "ring" were magically projected out into the portions of the visual field which it represents in the brain (as was done for the V2 strips), it would appear as a vertical stripe centered at or near the fixation of gaze. However, since the retinotopic specificity of MT shows a certain amount of organizational noise, this geometrical exercise may be more fanciful in MT than it is in V2.

Differences between cortical laminae have been commonly associated with differences in input and output connections. Thus, an 
examination of the laminar specialization of the otherwise radial topographies may suggest differences in afferent and efferent connections with other cortical areas. In the absence of tract tracing data the following suggestions can only be speculative, but they may serve to guide such future studies of intracortical connections.

In Aotus MT there is a sort of bisected radial topography in which the regions of high metabolic capacity overlie highly myelinated regions, and in which the converse also holds. One possible interpretation is that there are topographical inhomogeneities in the distribution of highly active, myelinated fibers coming up through the lower layers which would then quite naturally produce a similar pattern of metabolic activity in the layer of termination (layer 4) and above. Since MT receives major input from striate (see Weller and Kaas, 1982, for a review), and since specific connections have been shown between like regions of high and low cytochrome oxidase activity in V1 and V2 (Livingstone and Hubel, 1984), it is not farfetched to propose that regions of high and low cytochrome oxidase activity in striate and MT are similarly interrelated. By this model, the dark cytochrome oxidase spots in striate layer 4 would project to the darker regions in the central MT oval, and the lighter interspot regions of striate 4 would project to the lighter regions in the MT oval. A similar relationship might also hold for the projection from striate layer 6 to MT.

Support for this idea comes from both tract tracing data and comparative studies. First, the projection from striate cortex to MT is in fact patchy in MT (Montero, 1980; Lin, et al., 1982). Second, in two normal miacaques we have looked for cytochrome oxidase patterns in MT which are homologous to those we report here for Aotus. In the macaque, we found only a very faint darkening in the upper layers of MT; it appears almost negligible relative to the robust MT patterns in Aotus. However, this difference between species would also be predicted from the wiring model discussed above, since in the macaque the cytochrome oxidase blobs are either quite pale or absent from the layers which project to MT (that is, lamina $4 \mathrm{~B}$ and 6 ).

Laminar differences between the V2 strips in Aotus and those in other species are also interesting. In Aotus, the V2 strips disappear in the lower layers, but in the closely related squirrel monkey (Saimiri sciureus) the V2 strips are highest in contrast and most clearly defined in the lower layers (Tootell et al., 1983a). Since the thin-thick stripe distinction is also most obvious in the lower layers (Tootell et al., 1983a), this may explain why the distinction between thin and thick stripes is less obvious in Aotus than in other species. Since the contrast of the V2 strips is also greatest in the lower layers, this may partially explain why the contrast of the $\mathrm{V} 2$ strips in any layer is lower in Aotus than in the closely related Saimiri.

In the squirrcl monkcy, it has been shown that the pulvinar projects prominently to layer 5 of the (wide) V2 strips (Livingstone and Hubel, 1982). Since high cytochrome oxidase activity often marks regions of thalamocortical input (Livingstone and Hubel, 1982), the disappearance of the V2 strips in the lower layers of Aotus may signal a diminished (or conceivably a less active) projection from the pulvinar to the V2 strips in this species.

In studies preceding the discovery of the V2 strips, "fingers of degeneration" were reported in primate V2 running roughly perpendicular to the V1-V2 border following corpus callosum section (Newsome and Allman, 1980). Since the topography of these "fingers" corresponds roughly with that of the V2 strips (especially the thin strips), it is possible that $\mathrm{V} 2$ in each hemisphere is connected via callosal inputs which terminate either on the strips, or conceivably in the interstrip regions.

In general, we have found close correspondence between electrophysiologically defined cortical areas in Aotus and those described here using enzymatic staining methods. In a few specific instances, however, this correspondence breaks down. The first report of a discrepancy between cytochrome oxidase architecture and electrophysiological areas was that V2 strips in the macaque were confined to the binocular portions of the visual field (Tootell et al., 1983a), although the electrophysiological maps of V2 include a representation of both monocular and binocular regions (Gattas et al., 1981). The anatomical relationship between the monocular segment and the V2 strips can be defined precisely in the macaque because this genus has ocular dominance strips in the binocular, but not in the monocular, regions of the visual field. In Aotus, the electrophysiological maps also contain a representation of the monocular segment (Allman and Kass, 1974a), and in this species we also see a consistent strip-free gap adjacent to peripheral (presumably monocular) striate cortex. However, we have no direct anatomical evidence demonstrating a confinement of the V2 strips to the binocular visual field in Aotus.

Another area in which the details of the cytochrome oxidase topography are at variance with previous area boundaries is in Aotus MT. In this area the representation of the vertical meridian has been often described as coincident with the outer boundary of the region of high myelination. This myelin boundary is in turn topographically in register with the outer boundary of the central oval of high cytochrome oxidase activity in the middle and upper layers. However, surrounding all of these borders is a thin, dark ring of high enzyme activity. By current maps the ring would clearly lie beyond MT in surrounding area DL. However, the ring stains darkly for cytochrome oxidase, as does MT (and unlike DL). (It is for this reason that we have included the ring in our discussion of MT enzyme topography rather than DL topography.) Because the anatomy here is so specific, it may be worth considering whether the borders of "MT" should be expanded to include the ring (and perhaps the light-staining area between the central oval and the ring). It is not clear that the resolution of previous mapping measures was adequate to unambiguously exclude the ring from lying on (rather than surrounding and beyond) the representation of the vertical meridian.

In order to sensibly assign the ring region to either $M T$ or $D L$, it would be worthwhile to know more about the electrophysiology and the connections of this region. Detailed retinotopic maps would clarify receptive field positions in the ring relative to the vertical meridian. It is also known that cells in MT are almost uniformly direction selective: are cells within the MT ring also direction selective? Cortical area boundaries are also based on differences in intracortical connections: it would thus be useful to find out whether the afferent and efferent connections of the ring resemble those of MT or those of DL.

One of the most interesting of the mapping quandaries posed by the present data occurs in area VPIVA. The size and position of the enzymatic patterns anterior to ventral V2 place them roughly within the borders of electrophysiologically defined VP plus VA. However, there is enough uncertainty in this extrapolation that we describe the patterns as being in VPNA, meaning VP and perhaps VA. In order to resolve this ambiguity a within-hemisphere double label experiment is needed in which the cytochrome oxidase patterns can be compared to a label of the vertical meridian (by retinotopic 2deoxyglucose mapping, by degeneration stain after callosal section, or by electrophysiological mapping and marking). If it can in fact be established that the enzymatic topography of VP/NA extends over areas VP plus VA, this would be a highly unusual "area" indeed, since the "single" area would contain a mirror-image, duplicated representation of the visual field.

Double label (or physiological correlation) experiments would also clarify the significance of the cytochrome oxidase topography in auditory cortex. Pertinent questions here relate to the physiology and connections of these areas. For instance, how is the enormous variability of the cytochrome oxidase topography to be resolved with a horizontal tonotopic organization which (at least in previously published maps) looks much more orderly? Exactly which auditory areas are included in the regions of high enzymatic activity, and what are the connectional differences between areas of high and low cytochrome oxidase activity?

It has also been reported that auditory areas have relatively higher 
levels of 2-deoxyglucose uptake, at least in the rat (Sokoloff et al., 1977). Thus, it would nut be surprising if neural activity in area A were higher in the areas of increased cytochrome oxidase staining.

One advantage of defining auditory cortex on the basis of enzymatic rather than myelin or Nissl criteria is that the enzymatic patterns are many times higher in contrast and sharper in detail than the Nissl or myelin patterns. However, as discussed above, the exact relation of the enzymatic patterns to tonotopic and connectional variables needs to be defined before the enzymatic patterns can be of use.

Similar kinds of experiments are also called for in area 3b: again we find the overall topography of an area to be substantially different from that previously described. The area thus needs to be remapped (both physiologically and connectionally) relative to the anatomical topography in the same tissue. Although microelectrode maps have already been matched to Nissl boundaries of apparently singular dorsoventral $3 b$ strips, it may be that the higher contrast and more definite borders of the cytochrome oxidase stain will allow an anatomical clarification of the physiological duplication of skin surfaces which have previously been ascribed to areas $3 b$ and 1 (e.g., Merzenich et al., 1978).

Electrophysiological mapping studies would also be of great interest in examining the anteroventral leg of area $3 \mathrm{~b}$, since this area has been mapped in only a very preliminary way so far. The mouth and lower face appear to be mapped in this general region $(\mathrm{J} . \mathrm{H}$. Kaas, personal communication), but these preliminary data need to be related more exactly to the anatomical boundaries of area $3 \mathrm{~b}$ in the region.

In a comparison of electrophysiologically mapped extrastriate areas between the only two well mapped monkey genera (Macaca and Aotus), a number of discrepancies arise. Areas which occur in one genus have no apparent homologue in the other, and vice versa. Allman and Kaas $(1974 b, 1975)$ have proposed that different extrastriate areas may evolve as a result of mutant duplications and/ or alterations of existing visual areas in a given species. This implies that higher tier areas may be genetically more labile (and therefore more variable) than more primary visual areas. This idea is supported by our own data: progressively higher tier areas have increasingly more variable cytochrome oxidase topographies. The topography of V1 was quite constant across the 14 hemispheres we examined, V2 was more variable, and MT and VA/VP were more variable still. The logical limit of variability is reached in area DX, in which a distinctive, common pattern was seen in two animals (4 hemispheres), a different but vaguely related pattern was seen in two other animals, and little or no pattern was seen in another two animals. It is not clear how these individual differences are related to possible differences in karyotype within a single apparent species.

\section{References}

Allman, J. M., and J. H. Kaas (1971) A representation of the visual field in the caudal third of the middle temporal gyrus of the owl monkey (Aotus trivirgatus). Brain Res. 31: 84-105.

Allman, J. M., and J. H. Kaas (1974d) The oryarization of the second visual area (VII) in the owl monkey: A second order transformation of the visual hemifield. Brain Res. 76: 247-265.

Allman, J. M., and J. H. Kaas (1974b) A crescent-shaped cortical visual area surrounding the middle temporal area (MT) in the owl monkey (Aotus trivirgatus). Brain Res. 81: 199-213.

Allman, J. M., and J. H. Kaas (1975) The dorsomedial cortical visual area: A third tier area in the occipital lobe of the owl monkey (Aotus trivirgatus). Brain Res. 100: 473-487.

Allman, J. M., J. F. Baker, W. T. Newsome, and S. E. Petersen (1981) Visual topography and function: Cortical visual areas in the owl monkey. In Cortical Sensory Organization. Vol. 2: Multiple Visual Areas, C. N. Woolsey, ed., pp. 171-185, Humana Press, Clifton, NJ.

Carroll, E., and M. Wong-Riley (1982) Light and EM analysis of cytochrome oxidase-rich zones in the striate cortex of squirrel monkeys. Soc. Neurosci. Abstr. 8: 706.
Fitzpatrick, D., K. Itoh, and I. T. Diamond (1983) The laminar organization of the lateral geniculate body and the striate cortex in the squirrel monkey (Saimiri sciureus). J. Neurosci. 3: 673-702.

Gattas, R., C. G. Gross, and J. H. Sandell (1981) Visual topography of V2 in the macaque. J. Comp. Neurol. 201: 519-539.

Graybiel, A. M., and C. W. Ragsdale, Jr. (1982) Pseudocholinesterase staining in the primary visual pathway of the macaque monkey. Nature 299: 439-442.

Hendrickson, A. E., S. P. Hunt, and J. -Y. Wu (1981). Immunocytochemical localization of glutamic acid decarboxylase in monkey striate cortex. Nature 292: 605-607.

Horton, J. C. (1984) Cytochrome oxidase patches: A new cytoarchitectonic feature of monkey visual cortex. Philos. Trans. R. Soc. Lond. (Biol.) 304. 199-253.

Horton, J. C., and D. H. Hubel (1981) Regular patchy distribution of cytochrome oxidase staining in primary visual cortex of macaque monkeys. Nature 292: 7622-764.

Imig, T. J., M. A. Ruggero, L. M. Kitzes, E. Javel, and J. F. Brugge (1977) Organization of auditory cortex in the owl monkey (Aotus trivirgatus). J. Comp. Neurol. 171: 111-128.

Kaas, J. H., C. S. Lin, and V. A. Casagrande (1976) The relay of ipsilateral and contralateral retinal input from the lateral geniculate nucleus to striate cortex in the owl monkey: A transneuronal transport study. Brain Res. 106: 371-378.

Lin, C. S., R. E. Weller, and J. H. Kaas (1982) Cortical connections of striate cortex in the owl monkey. J. Comp. Neurol. 211: 165-176.

Livingstone, M. S., and D. H. Hubel (1982) Thalamic inputs to cytochrome oxidase-rich regions in monkey visual cortex. Proc. Natl. Acad. Sci. U. S. A. $79: 6098-6101$.

Livingstone, M. S., and D. H. Hubel (1984) Anatomy and physiology of a color system in the primate visual cortex. J. Neurosci. 4: 309-356.

Merzenich, M. M., M. Sur, R. J. Nelson, and J. H. Kaas (1981) The organization of the SI cortex: Multiple representation of the body in primates. In Cortical Sensory Organization. Vol. 1: Multiple Somatic Areas, C. N. Woolsey, ed., pp. 36-48, Humana Press, Clifton, NJ.

Montero, V. M. (1980) Patterns of connection from the striate cortex to cortical visual areas in the superior temporal sulcus of macaque and middle temporal gyrus of owl monkey. J. Comp. Neurol. 189: 45-59.

Newsome, W. T., and J. M. Allman (1980) Interhemispheric connections of visual cortex in the owl monkey, Aotus trivirgatus, and the bushbaby, Galago senegalensis. J. Comp. Neurol. 194: 209-233.

Rockland, K. S., and J. S. Lund (1983) Intrinsic laminar lattice connections in primate visual cortex. J. Comp. Neurol. 216: 303-318.

Rowe, M. H., L. A. Benevento, and M. Rezak (1978) Some observations on the patterns of segregated geniculate inputs to the visual cortex in New World primates: An autoradiographic study. Brain Res. 159: 371-378.

Sokoloff, L., M. Reivich, C. Kennedy, M. H. Des Rosiers, C. S. Patlak, K. D. Pettigrew, O. Sakurada and M. Shinohara (1977) The [C14] deoxyglucose method for measurement of local cerebral glucose utilization theory, procedure, and normal values in the conscious and anesthesized rat. J. Neurochem. 28: 897-916.

Tootell, R. B. H., and M. S. Silverman (1981) A comparison of cytochrome oxidase and deoxyglucose patterns in macaque visual cortex. Soc. Neurosci. Abstr., 7: 356 .

Tootcll, R. B. H., M. S. Silverman, E. Switkes, and R. L. De Valois (1982) Organization of cortical modules. Soc. Neurosci. Abstr. 8: 707.

Tootell, R. B. H., M. S. Silverman, R. L. De Valois, and G. H. Jacobs (1983a) Functional organization of the second cortical visual area in the primate. Science 220: 737-739.

Tootell, R. B. H., M. S. Silverman, and R. L. De Valois (1983b) Topography of cytochrome oxidase activity in the owl monkey. Soc. Neurosci. Abstr. 9: 477.

Van Essen, D. C., J. H. R. Maunsell, and J. L. Bixby (1981) The middle temporal visual area in the macaque: Myeloarchitecture, connections, functional properties and topographic organization. J. Comp. Neurol. 199: 293-326.

Weller, R. E., and J. H. Kaas (1982) Cortical and subcortical connections of visual cortex in primates. In Cortical Sensory Organization. Vol. 2: Multiple Visual Areas, C. N. Woolsey, ed., pp. 121 155, Humana Press, Clifton, NJ.

Wong-Riley, M. T. T. (1979) Changes in the visual system of monocularly sutured or enucleated cats demonstrable with cytoch rome oxidase histochemistry. Brain Res. 171: 11-28. 